\title{
Caracterización fisiológica del enraizamiento in vitro de Eucalyptus nitens y Eucalyptus globulus
}

\section{Physiological characterization of in vitro rooting of Eucalyptus nitens and Eucalyptus globulus}

\author{
María Paz Jofré ${ }^{*}$, Darcy Ríos ${ }^{1}$, José Becerra $^{2}$ \& Manuel Sánchez-Olate ${ }^{1}$ \\ ${ }^{1}$ Laboratorio de Cultivo de Tejidos Vegetales, Facultad de Ciencias Forestales y Centro de Biotecnología, Universidad de \\ Concepción, Casilla 160-D, Correo B, Concepción, Chile. \\ 'Laboratorio de Química de Recursos Naturales, Facultad de Ciencias Naturales y Oceanográficas, Universidad de \\ Concepción, Casilla 160-C, Concepción, Chile. \\ *majofre@udec.cl
}

\section{RESUMEN}

El entendimiento de los cambios bioquímicos que ocurren durante las distintas fases del enraizamiento hace posible obtener mayor porcentaje de enraizamiento de microbrotes en especies leñosas. Para diferenciar ambas especies y encontrar un marcador de la capacidad rizogénica, en el presente estudio se pretende caracterizar perfiles cinéticos de Ácido Indol Acético (AIA), Fenoles totales y Carbohidratos totales. Para esto se evaluaron microtallos de las especies Eucalyptus nitens (H. Deane \& Maiden) Maiden y Eucalyptus globulus Labill. las cuales fueron tratadas con el método de la inducción lenta del enraizamiento en medio de cultivo Murashige \& Skoog (1962) (MS) con las sales a la mitad, 30 g/L de sacarosa, suplementado con Ácido Naftalen Acético (ANA) durante 7 días en oscuridad. Posteriormente transferidos a sustrato vermiculita estéril con riego de medio de cultivo MS sin reguladores del crecimiento. Se recolectó material vegetal los días 0, 2, 7 y 20 días desde la inducción, se tomaron muestras de base de estacas para los análisis fitoquímicos. El AIA en los días 0 y 2 presentó altos valores que disminuyeron drásticamente durante las siguientes fases del enraizamiento días 7 y 20. Los compuestos fenólicos presentaron diferencias entre las dos especies en el tiempo evaluado, con valores significativamente más altos en Eucalyptus nitens (136 ug/gPS) que en E. globulus (29 ug/gPS). Los carbohidratos totales no mostraron diferencias entre las especies y se mantienen altos durante todo el tiempo en las dos especies de Eucalyptus. De acuerdo a lo obtenido, los compuestos fenólicos son buenos marcadores de la capacidad de enraizamiento adventicio ya que se diferenciaron significativamente en las dos especies de Eucalyptus.

Palabras clave: Flavonoides, Acido Indol Acético, enraizamiento adventicio.

\begin{abstract}
The understanding of the biochemical changes that occur during the different phases of rooting makes it possible to obtain higher percentage microshoots rooting in woody species. To differentiate the two species and find a marker rhizogenic capacity in the present study is to characterize kinetic profile of Indole Acetic Acid content (AIA), total phenols and total carbohydrates. To this microshoots species Eucalyptus nitens (H. Deane \& Maiden) Maiden and Eucalyptus globulus Labill. which were evaluated were treated with the method of the slow induction of rooting on Murashige \& Skoog (1962) (MS) with the salts in half, supplemented with auxin naphthaleneacetic acid (NAA) for 7 days in darkness. Subsequently transferred to sterile vermiculite substrate irrigated crop MS medium without hormones. Plant materials on days 0, 2, 7 and 20 days from the induction manifolds, core samples were taken cuttings for analysis phytochemicals. The AIA on days 0 and 2 , showed high values decreased dramatically during the next phases of rooting days 7 and 20. Phenolic compounds showed differences between the two species evaluated in time, with significantly higher values in Eucalyptus nitens (136 ug/gPS) in E. globulus (29 ug/gPS), total carbohydrates showed no differences between species and stay high all the time The AIA is essential for induction of rooting and is not subsequently required, as carbohydrates are not candidates for markers rhizogenic capacity not because they differ in the two species of Eucalyptus. According to the obtained phenolic compounds are good markers of the capacity of adventitious rooting since differed significantly in the two species of Eucalyptus.
\end{abstract}

KEYwords: Indol Acetic Acid, adventitious rooting, phenolic compounds. 


\section{INTRODUCCIÓN}

Uno de los grandes problemas que presentan las especies del género Eucalyptus durante el proceso productivo de propagación vegetativa corresponde a la pérdida de la competencia rizogénica en tejidos adultos, por lo que es necesario el desarrollo de métodos de clonación eficientes. En la actualidad existen pocos estudios referentes a optimizar los mecanismos de propagación en Eucalyptus nitens (H. Deane \& Maiden) Maiden, por lo que el cultivo in vitro es una alternativa de propagación para obtener clones superiores con características deseadas para el desarrollo comercial que ha sido exitosa en Eucalyptus (Campbell et al. 2003, Delaporte \& Sedgley 2004, Gaspar et al. 2005). El cultivo in vitro es una herramienta rápida de multiplicación que permite obtener individuos libres de patógenos con estabilidad genética, optimización de espacio y tiempo, y no depende de los periodos de producción (Badr \& Desjardins 2007). Sin embargo dentro de sus desventajas, en Eucalyptus se encuentra el bajo porcentaje de enraizamiento y sobrevivencia de los explantes (Popsilová et al. 2000, Kozai \& Kubota 2005), comportamiento también observado en condiciones in vivo (Fett-Neto et al. 2001).

El enraizamiento adventicio es una respuesta multifactorial que finalmente conduce a la formación de una raíz a partir del tallo. Ocurre en distintas fases que se caracterizan por presentar condiciones y requerimientos bioquímicos diferentes (Caboni et al. 1997, Kevers et al. 1997, De Klerk et al. 1999, Schwambach et al. 2008). En este sentido el cultivo in vitro presenta ventajas para el estudio de estas fases ya que se facilita la adición de auxinas y otros compuestos, inhibe la degradación microbiana, permite la adición de nutrientes inorgánicos y carbohidratos y estimula experimentos a pequeña escala, permitiendo hacer avances en experimentos en métodos moleculares (De Klerk et al. 1999).

Durante estas fases, cambios de factores físicos y químicos influencian la inducción y expresión de raíces adventicias, como fotoperiodo, contenido de AIA, compuestos fenólicos y poliaminas, entre otros (Martinez Pastur et al 2003, Arena et al. 2005). El entendimiento de estos factores bioquímicos hace posible mejorar el enraizamiento in vitro en especies leñosas (Martinez l.c.).

El cofactor estimulador por excelencia del enraizamiento adventicio es la auxina AIA (Fett-Neto et al. 2001, Davies 2004, Woodward \& Bartel 2005). El rol fundamental de AIA se basa en que es el único químico que consistentemente estimula el enraizamiento de forma directa, ya sea por fuente endógena o por abastecimiento exógeno, el transporte de auxinas endógeno es principalmente basipétalo y está de acuerdo a la formación polar de la raíz. Por otro lado, al inhibir el transporte de auxinas se bloquea la formación de la raíz (Assis et al 2004). Además existe evidencia bioquímica y genética debido a la identificación de receptores de auxinas asociados con la habilidad de enraizamiento (Woodward et al. 2005).

En especies de Eucalyptus con alto porcentaje de enraizamiento como E. saligna $\mathrm{Sm}$. es suficiente con AIA endógeno para estimular el enraizamiento (Schwambach et al. 2008). En cambio, en E. globulus Labill. que presenta menor porcentaje de enraizamiento es necesaria una aplicación exógena (Martellet \& Fett-Neto 2005).

Sin embargo, existen especies en las que es requerida una aplicación exógena de auxina para estimular la rizogénesis (Díaz-Sala et al. 1996). Por ejemplo en renovales apicales se reduce la disponibilidad de AIA, por lo que una aplicación exógena incrementa el número de raíces formadas (Nordstrom et al. 1991, Liu \& Reid 1992). El Ácido Indol Butírico (AIB) es un precursor de AIA que dirige la expansión celular en tejidos específicos como pelos radiculares (Strader et al. 2010). Aplicado exógenamente AIB actúa a tres niveles: induce el movimiento basipétalo de AIA endógeno (Teale et al. 2006), su conversión en AIA (Nordstrom et al. 1991, Ludwig-Müller 2000, Bartel et al. 2001) y produce mayor estabilidad química al mantener niveles adecuados de AIA libre (Ludwig- Müller 2000).

El rol de las auxinas en el enraizamiento es directo (De Klerk et al. 1999), sin embargo, los demás factores que controlan el enraizamiento lo hacen a través de la homeostasis de AIA haciéndola más o menos disponible. En este sentido existe una coordinación bioquímica del proceso rizogénico controlada por el contenido de peroxidasas totales y compuestos fenólicos (Calderón Baltierra 1994, Pacheco et al. 1995, Caboni et al. 1997). Los compuestos fenólicos son factores de defensa contra varios tipos de estres causados por patógenos, ambiente adverso o herida (Solar et al. 2006). La relación entre los fenoles y la rizogénesis inducida por auxina puede ser muy variada, en algunas ocasiones actúan como cofactores rizogénicos, apoyando la fase de iniciación radicular y en otras dependiendo de la calidad y cantidad en que se encuentren pueden influir sobre la oxidación de la auxina (Latsague \& Lara 2003), impidiendo la formación de raíces adventicias. Además dentro de los compuestos fenólicos importantes en la regulación del proceso rizogénico se encuentran los flavonoles (Koes et al. 2005, Taylor \& Grotewold 2005), que inhiben el enraizamiento al bloquear su transporte (Peer et al. 2004, Murphy et al. 2000).

En el presente estudio se pretende evaluar algunos componentes bioquímicos del enraizamiento para establecer un marcador de la capacidad rizogénica de dos especies de Eucalyptus, uno de mayor capacidad E. globulus y otro de baja capacidad como E. nitens.

\section{MATERIALES Y MÉTODOS}

Material VegetaL

Como explantes se usaron vitroplantas de clones de Eucalyptus nitens y Eucalyptus globulus pertenecientes a 
vivero Forestal Mininco S.A., previamente multiplicadas y elongadas, para inducir el proceso de enraizamiento en el laboratorio de Cultivo de Tejidos Vegetales, Centro de Biotecnología de la Universidad de Concepción.

\section{INDUCCIÓN DEL ENRAIZAMIENTO}

Las vitroplantas se enraizaron por el método de "Inducción lenta", que consiste en la exposición a medio sólido MS con los macroelementos a la mitad de su concentración, suplementado con $3 \mathrm{mg} / \mathrm{L}$ de NAA durante 7 días en oscuridad en cámara de cultivo a $20^{\circ} \mathrm{C}$. Posteriormente se transfirieron a sustrato de vermiculita estéril tapado con plástico y se regaron cada dos días con solución nutritiva de medio MS líquido hasta el día 20 desde la inducción. Se colectó material consistente en base de tallos los días $0,2,7$ y 20 para los análisis fitoquímicos correspondientes.

\section{DeTERMINACIÓN DEL CONTENIDO DE AIA}

De los microtallos recolectados en los tiempos indicados como los tratamientos, se extrajo AIA con Metanol siguiendo el procedimiento descrito por Valenzuela et al. (1998). Se extrajo la fase orgánica y la fase acuosa que contienen la hormona libre y conjugada, respectivamente. Los extractos se resuspendieron en metanol absoluto y se analizaron a través de HPLC Shimadzu Japón Kyoto, anexado a un computador por el programa D 7000 HPLC ensamblado a una bomba D7200 y el detector UV L/7400.

Se separaron por HPLC RP-18 fase reversa 2504mm (5 um) Bio Rad en columna Lychrospher 100 con dosificación $0,8 \mathrm{ml} / \mathrm{min}$. La identificación fue obtenida por comparación con una curva patrón estándar de AIA Sigma.

\section{Determinación DE FENOLES TOTALES}

El contenido de fenoles totales se estimó como equivalentes de scido gálico de acuerdo al método modificado de Gutiérrez et al. (2008), que utiliza el reactivo Folin Ciocalteu (agente oxidante) formando complejos con los fenoles cromógenos de coloración proporcional al número de hidroxilos presentes en éstos. Se cuantificaron por espectrofotometría en comparación a una curva de calibración.

\section{DETERMINACIÓN DE CARBOHIDRATOS SOLUBLES}

Se determinó por el Método del Fenol sulfúrico por espectrofotometría en comparación a una curva patrón de glucosa.

\section{ANÁLISIS ESTADÍSTICO}

El análisis estadístico fue realizado con Modelos Estadísticos Lineales. Para el análisis de concentración de compuestos fenólicos, AIA y carbohidratos, se realizó un análisis de mediciones repetidas en el tiempo o también conocido como análisis longitudinal. Este análisis permite identificar tanto el efecto medio del tratamiento (especie), el efecto del tiempo (tiempo), y la interacción tiempo por tratamiento, que corresponden a las tres hipótesis evaluadas, y que por lo tanto generan tres valores p. La estimación de parámetros se realizó por máxima verosimilitud restringida (REML). La selección del modelo se hizo con el criterio de información de Akaike (menor es mejor). Los modelos fueron estimados utilizando SAS versión 9.3 PROCMIXED (SAS Institute, 2006). Las comparaciones por pares entre asociaciones de especies fueron probados con los LSMEANS ajustados $(\alpha=0,05)$ (Littell et al. 2006).

\section{RESULTADOS Y DISCUSIÓN}

El enraizamiento in vitro es la etapa final del proceso productivo de micropropagación que precede a una etapa de multiplicación con subcultivos periódicos en medio rico en citoquinina y elongación de las vitroplantas en un medio rico en auxinas. En la Figura 1 es posible observar microtallos en estado de proliferación (a) y etapa de elongación (b). Estas etapas son claves en los resultados de enraizamiento que se quiere obtener. Ríos et al. (2002) señalaron que el proceso de enraizamiento está influenciado por diversos factores tales como el suministro de oxígeno, temperatura, sacarosa, potencial estomático, $\mathrm{pH}$ y nutrientes minerales que dependen básicamente de las condiciones físicas de cultivo (Assis et al. 2001). Otros factores importantes a considerar en la fase de enraizamiento son vigor, estado sanitario, longitud y diámetro de los microtallos, ya que diversos autores señalan altos porcentajes de enraizamiento a mayor diámetro y vigor de los explantos inducidos (Ríos et al. 2002). Por otro lado, en E. urophylla S.T. Blake y E. grandis W. Hill, se ha relacionado el contenido de AIA con el número de subcultivos a mayor frecuencia menor capacidad rizogénica (Mankessi et al. 2009).

\section{DETERMINACIÓN DEL CONTENIDO DE AIA}

En la Tabla I se observa presencia de AIA en los dos primeros días desde la inducción del enraizamiento. Este tiempo corresponde a la etapa señalada como Inducción (Kevers et al. 1997, De Klerk et al. 1999) y este nivel de auxina es necesario para producir la diferenciación seguida de un proceso de desdiferenciación en la inducción temprana (antes de $24 \mathrm{~h}$ ). Las auxinas en esta etapa se transportan y canalizan a células diana. Allí actúan principalmente mediante la proteólisis selectiva de proteínas receptoras RIT1 respuesta inhibidor del transporte 1) y ABP1 (Auxina-Binding Protein 1) (Schwambach et al. 2008). Posteriormente producen aflojamiento de la pared celular. La presencia de AIA es esencial en esta etapa ya que controla la expresión génica del enraizamiento a través de factores de respuesta a auxinas (Schwambach et al. 2008). 
Flores (2009) en Vigna radiata (L.) R. Wilczek encontró el más alto nivel de AIA al día 3 post inducción del enraizamiento y una disminución al día 6 .

En la Tabla I se observa una significativa disminución de AIA el día 7 desde la inducción (0\%), lo que podría corresponder a la fase de iniciación del enraizamiento en que ocurren sucesivas divisiones celulares que darán origen al primordio radicular. Los niveles de las auxinas libres permanecen altos durante la fase de inducción del enraizamiento y decrecen justo al comenzar la fase de iniciación (Nag et al. 2001). Después del establecimiento de la raíz, nuevos reguladores del crecimiento tienen lugar, las auxinas son requeridas en concentraciones más bajas sólo para el mantenimiento de estados diferenciados y citoquininas son necesarias para la diferenciación de los tejidos de la raíz (Schwambach et al. 2008). En Eucalyptus globulus se observan cambios a partir del día 7 (Calderón Baltierra 1994) en el proceso de enraizamiento in vitro: desorganización celular de la base de microtallos, en el parénquima cortical con prominentes núcleos (Reinhard et al. 2000). Además, en esa fase los niveles de conjugados auxínicos se incrementan (Davies 2004). En contraste, las peroxidasas muestran baja actividad de AIA-ox durante la inducción, seguida de un aumento durante la iniciación (Nag et al. 2001, Qaddoury \& Amsa 2004). Estas peroxidasas apoplásticas acidificarían las inmediaciones del tejido destino como señalización de membrana. Las auxinas a través del bombeo de $\mathrm{H}$ producen plasticidad de la pared celular para la elongación celular y crecimiento durante la etapa de iniciación (Robert-Klever et al. 2003).

TABLA I. Contenido de AIA libre en explantes inducidos a enraizar in vitro. Solo hay presencia de niveles detectables de AIA los días 0 y 2 y vuelve a aparecer el día 20 en que ya es posible observar raíz primordial.

TABLE I. Content of free IAA in root explants induced in vitro. There's only presence of detectable levels of AIA on days 0 and 2 and reappears on the 20th that it is possible to observe primary root.

\begin{tabular}{ccc}
\hline Días De RECOLECTA & $\begin{array}{c}\text { Conc. AIA ug/gPS } \\
\text { E. nitens }\end{array}$ & $\begin{array}{c}\text { Conc. AIA ug/gPS } \\
\text { E. globulus }\end{array}$ \\
\hline 0 & $28 \mathrm{a}$ & $32 \mathrm{a}$ \\
2 & $22 \mathrm{a}$ & $25 \mathrm{a}$ \\
7 & 0 & 0 \\
20 & $8 \mathrm{~b}$ & $9 \mathrm{~b}$ \\
\hline
\end{tabular}
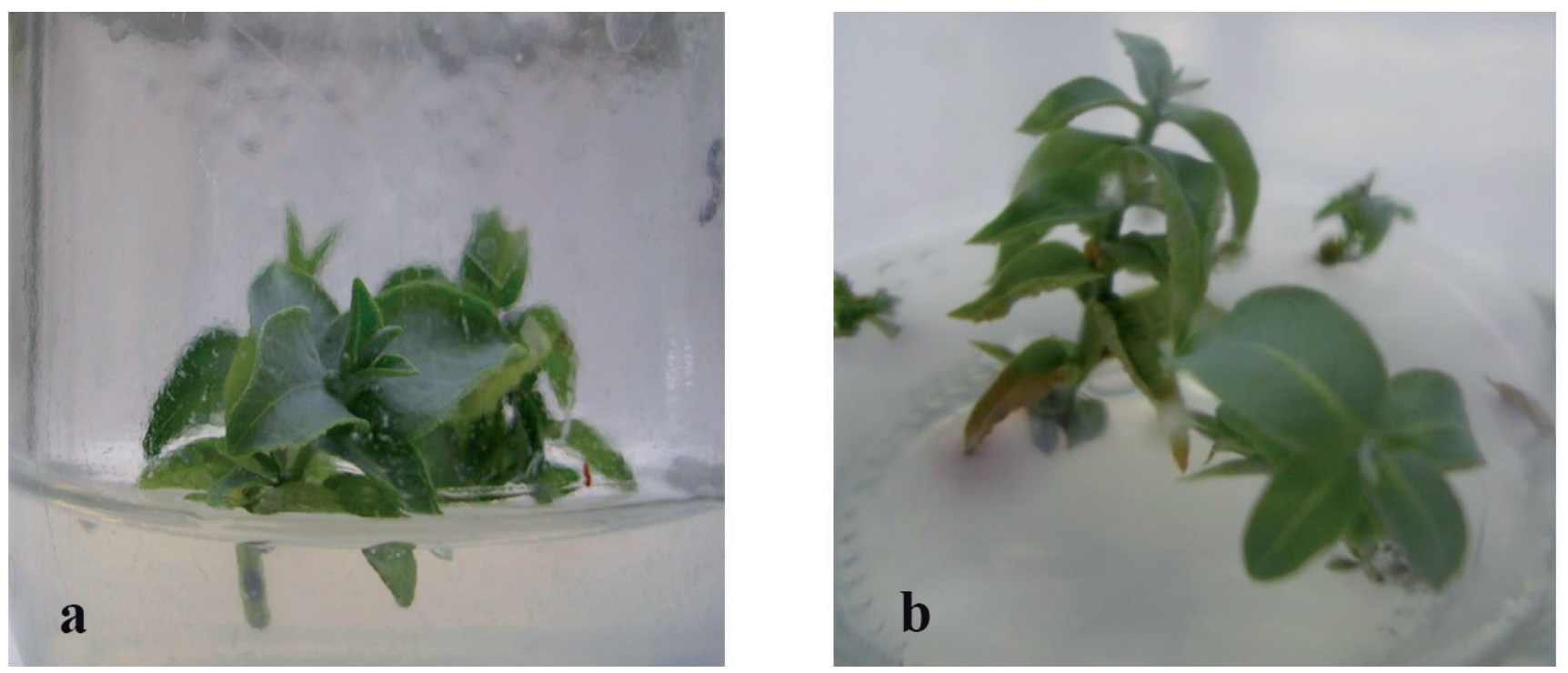

Figura 1: Microtallos creciendo in vitro (a) en multiplicación y (b) en elongación de $2 \mathrm{~cm}$ de longitud.

FigURE 1: Eucalyptus nitens microshoots growing in vitro (a) proliferation medium and (b) elongation medium of $2 \mathrm{~cm}$ in length. 
El día 20 post inducción en la Tabla I se observa un leve aumento en el nivel de AIA en ambas especies, esta etapa in vitro es la de expresión en que ya se han formado los primordios radiculares (Kevers et al. 1997, De Klerk et al. 1999). En la Fig. 2A se observa un tallo de E. nitens con la aparición de las primeras raíces, la formación del meristema radicular es directa ya que no se produce callo en la base del tallo. Lo que es ventajoso, ya que la formación de este tejido puede interferir en la conexión vascular que se establece entre las nuevas raíces y el explanto original (Martínez Pastur et al. 2005, San José et al. 2012). Esta etapa en que AIA vuelve a aparecer probablemente para la diferenciación de tejidos vasculares e independencia del primordio radicular el día 20, es semejante a la encontrada en el enraizamiento in vitro de otras especies, como manzano (Naija et al. 2008) y castaño (Ballester et al. 2009). Y en Eucalyptus globulus, a partir del día 18 (Calderón et al. 1994). Sin embargo, el tiempo requerido para la formación de las raíces adventicias varía con la especie, se observó al cabo de 8 días en el caso de Camellia japonica L., 5 días en Malus (Naija et al. 2008). En híbridos de E. globulus x maidennii se encontraron fases similares: inducción día 5 , iniciación entre los días 5 y 15 y elongación después de los 15 días (Schwambach et al. 2008).
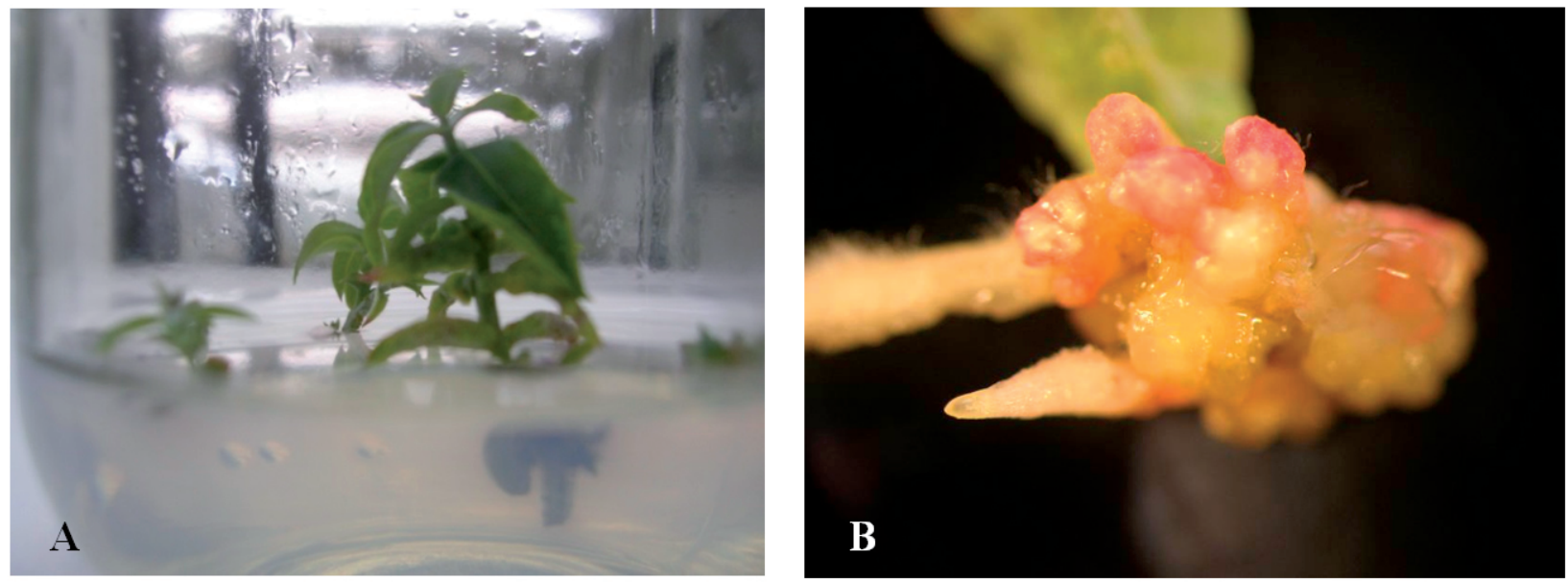

Figura 2. Enraizamiento in vitro de Eucalyptus nitens. A) Microtallo con formación de primordio radicular en la base del tallo a los 13 días desde la inducción. B) Primordio radicular mostrando dos raíces principales después de 20 días desde la inducción.

Figure 2. In vitro rooting of Eucalyptus nitens. A) Microshoot with incipient formation of root primordia after of thirteen days of induction. B) Root primordium with two roots after twenty days of induction.

DETERMINACIÓN DE COMPUESTOS FENÓLICOS

Al analizar los fenoles totales en ambas especies de Eucalyptus como lo muestra la Tabla II, se observa que Eucalyptus nitens presenta concentraciones significativamente mayores que Eucalyptus globulus. En las dos especies la dinámica es la misma con valores más altos durante la fase de inducción los dos primeros días y una disminución el día 7 y 20.

Un comportamiento similar ha sido observado en Eucalyptus en que genotipos con distinta habilidad para enraizar presentan altos niveles durante la fase de inducción del enraizamiento y decae durante la fase de iniciación, y se contradice con el contenido de AIA oxidasa, por lo que podría tratarse de inhibidores del enraizamiento (Caboni et al. 1997).

El elevado nivel de compuestos fenólicos en Eucalyptus nitens se puede relacionar con su baja capacidad de enraizamiento. Si éstos corresponden a monofenoles que actúan como inhibidores del proceso rizogénico reduciendo la concentración de AIA. Estos monofenoles como el ácido p-coumárico, ferúlico y siríngico promueven la descarboxilación de AIA activando a la enzima AIA oxidasa (De Klerk 2011).

En Nothofagus pumilio (Poepp. \& Endl.) Krasser se encontró que altas concentraciones de compuestos fenólicos solubles estarían induciendo la oxidación de la auxina aplicada al tratamiento de enraizamiento. Estos fenoles solubles puede pasar a ser inhibidor del enraizamiento dependiendo de la calidad y cantidad de éstos (Latsague \& Lara 2003).

Para Eucalyptus nitens no se trataría de polifenoles ya que éstos inducen el enraizamiento, como el ácido clorogénico, protocatéuchico y caféico, que suprimen 
la degradación de AIA debido a que activan la enzima polifenol oxidasa que los oxida a quinonas y no serían capaces de unirse ni estimular a la enzima AIA oxidasa (Ríos et al. 2005, De Klerk et al. 2011).

Por otro lado, la baja capacidad de enraizamiento de $E$. nitens y su alto contenido de compuestos fenólicos indica que podría tratarse de flavonoides inhibidores competitivos del enraizamiento, que inhiben el transporte polar de auxinas al unirse a proteínas PIN ya que su estructura química es similar al inhibidor endógeno ácido naftiltalámico NPA (Murphy et al. 2000, Brown et al. 2001, Peer et al. 2004).

\section{DETERMINACIÓN DE CARBOHIDRATOS TOTALES}

El contenido de Carbohidratos totales no presenta diferencias significativas durante los días evaluados en las dos especies. Como lo muestra la Tabla III, los niveles de carbohidratos presentes en todos los tiempos pueden estar indicando que solo están presentes para mantener funciones celulares constitutivas. Por otro lado, pueden indicar lo necesarios que son como fuente de energía, ya que durante el enraizamiento ocurren numerosos procesos biológicos como la biosíntesis de ácidos nucleicos, formación de nuevas enzimas y división celular. Varias enzimas aumentan su actividad como las respiratorias y amilasa (Fett-Neto et al. 2001). No todos los carbohidratos influyen en el enraizamiento adventicio, por ejemplo, en Arabidopsis, la glucosa, sacarosa y fructosa estimulan el enraizamiento adventicio, pero manosa y sorbitol no tienen efecto (Takahashi et al. 2003). La interacción de los carbohidratos con la señalización de los reguladores del crecimiento vegetal y su influencia en la modulación de la expresión génica (Geiss et al. 2009, Li et al. 2009) podrían determinar su papel en el proceso del enraizamiento adventicio a través de rutas aún no dilucidadas. Por otro lado, se ha encontrado que células que rodean los haces vasculares que sufren desdiferenciación y se hacen competentes para ser inducidas por auxinas las primeras $24 \mathrm{~h}$ de la inducción, acumulan almidón durante esta fase para ser utilizado en la fase de iniciación en que ocurren sucesivas divisiones celulares (De Klerk et al. 1999).

TABLA II. Contenido de fenoles totales en explantes inducidos a enraizar in vitro. La especie E. nitens presenta concentraciones significativamente más altas que E. globulus.

TABLE II. Content of total phenols in explants induced to root in vitro. The species $E$. nitens has significantly higher concentrations than $E$. globulus.

\begin{tabular}{ccc}
\hline DíAs DE RECOLECCIÓN & $\begin{array}{c}\text { Eucalyptus nitens } \\
\text { ug/gPS }\end{array}$ & $\begin{array}{c}\text { Eucalyptus globulus } \\
\text { ug/gPS }\end{array}$ \\
\hline 0 & $45 \mathrm{a}$ & $13 \mathrm{~b}$ \\
2 & $48 \mathrm{a}$ & $11 \mathrm{~b}$ \\
7 & $20 \mathrm{~b}$ & $9 \mathrm{~b}$ \\
20 & $51 \mathrm{a}$ & $8 \mathrm{~b}$ \\
\hline
\end{tabular}

TABLA III. Contenido de carbohidratos $(\mathrm{CH})$ totales en explantes inducidos a enraizar in vitro. No hay diferencias significativas entre las dos especies de Eucalyptus.

TABLE III. Content of carbohydrates $(\mathrm{CH})$ total explants induced to root in vitro. No significant differences between the two species of Eucalyptus.

\begin{tabular}{ccc}
\hline Días DE RECOLECCIÓN & $\begin{array}{c}\text { Conc. } \text { CH ug/gPS } \\
\text { E. nitens }\end{array}$ & $\begin{array}{c}\text { Conc. CH ug/gPS } \\
\text { E. globulus }\end{array}$ \\
\hline 0 & 406 & 501 \\
2 & 397 & 430 \\
7 & 270 & 420 \\
20 & 320 & 380 \\
\hline
\end{tabular}




\section{CONCLUSIONES}

La evaluación de los indicadores bioquímicos analizados para Eucalyptus nitens y Eucalyptus globulus que difieren en su capacidad de enraizamiento indica que:

- El AIA es fundamental en la fase de inducción del enraizamiento y no es requerido en alta concentración en la fase de iniciación. Sin embargo, no es un marcador de la capacidad de enraizamiento en Eucalyptus. ya que no presentó diferencias significativas entre las dos especies.

- El alto contenido de fenoles totales se relaciona con la baja capacidad de enraizamiento de $E$. nitens.

- El contenido de fenoles totales es un buen marcador de la capacidad de enraizamiento en Eucalytptus ya que presentó diferencias significativas entre las especies.

- El contenido de carbohidratos totales no es un marcador de la capacidad de enraizamiento en Eucalyptus ya que no presentó diferencias significativas entre las dos especies.

- Es necesario investigar más acerca de compuestos fenólicos específicos del tipo flavonoides para el entendimiento de un control negativo del proceso rizogénico.

\section{AGRADECIMIENTOS}

La realización de este estudio ha sido posible gracias a la colaboración de la empresa Forestal Mininco S.A. por su aporte en material vegetal; a la Comisión Nacional de Investigación Científica y Tecnológica, CONICYT, por beca estudio de María Paz Jofré, y a proyecto 1020F11 INNOVA Bío Bío, por financiamiento de Tesis en la que se enmarca este artículo.

\section{BIBLIOGRAFIA}

Arena, M.E., G. Martinez Pastur, M.P. Benavides \& N. Curvetto. 2005. Polyamines used in successive culture media improve in vitro rooting in Berberis buxifolia Lam. New Zealand Journal of Botany 43: 373-380.

Assis, T., A Fett-Neto \& A. Coulo. 2001 Current techniques and prospects for the clonal propagation of hardwoods with emphasis on Eucalyptus. Plantation Forest Biotechnology for the 21st Century, 2004: 303-333 ISBN: 81-7736-228318.

Badr, A. \& Y. Desjardins. 2007. Sugar uptake and metabolism in tissue cultured potato plantlets cultured in liquid medium. In: J.M. Santamaría \& Y. Desjardins (eds.), Proceedings II IS on Acclimatation and Establishment of Micropropagation Plants. Acta Horticulturae 748: 265-273.

Ballester, A., N, Vidal \& A.M. Vieitez. 2009. Developmental stages during in vitro rooting of hardwoods trees with juvenile and mature characteristics. In: K. Niemi \& C. Scagel (eds.). Adventitious Root Formation of Forest Trees and Horticultural Plants-from Genes to Applications Pp. 277-296. Research Signpost, Kerala, India.
Bartel, B., S. Leclere. M. Magidin \& B.K. Zolman. 2001. Inputs to the active indole- 3 -acetic acid pool: de novo synthesis, conjugate hydrolysis, and indole-3-butyric acid b-oxidation. Journal of Plant Growth Regulation 20: 198-216.

Brown, D., A. Rashotte, A. Murphy, J. Normanty, B. Tague, W. Peer, L. Taiz \& G. Muday. 2001. Flavonoids Act as Negative Regulators of Auxin Transport in Vivo in Arabidopsis. Plant physiology 126: 524-535.

Caboni, E., M.G. Tonelli, P. Lauri, P. Iacovacci, C. Kevers, C. Damiano \& T. Gaspar. 1997. Biochemical aspects of almond microcuttings related to in vitro rooting ability. Biologia Plantarum 19: 91-97.

Calderon Baltierra, X. 1994. Changes in peroxidase activity during root formation by Eucalyptus globulus shoot raised in vitro. Plant Peroxidase Newsletter 4: 27-29.

Campbell, M., A. Brunner, H. Jones \& S. Strauss. 2003. Forestry is fertile crescent the application of biotechnology to forest trees. Plant Biotechnology Journal 1: 141-154

DAVIES, P.J. 2004. Plant Hormones, Biosynthesis, Signal Transduction, Action. Edition $3^{\text {rd }}$. Kluwer Academic Publishers Netherlands. XIV, 802 pp.

De Klerk, G., W. Krieken \& J. De Jong. 1999. The formation of adventitious root: new concepts, new possibilities. In vitro cell development. Biology Plant 35: 189-199.

De Klerk, G., H. Guan, P. Huisman \& S. Marinova. 2011. Effects of phenolic compounds on adventitious root formation and oxidative decarboxylation of applied indoleacetic acid in Malus 'Jork 9'. Plant Growth Regulation 63: 175-185.

Delaporte, K. \& M. Sedgley. 2004. Breeding of Eucalyptus bud and flower lines. A report for the Rural Industries Research and Development Corporation. Clonal Propagation. Pp 22-33.

Diaz-Sala, C., K.W. Hutchison, W. Goldfarb \& M.S. Greenwood. 1996. Maturation related loss in rooting competence by loblolly pine stem cuttings: the rote of auxin transport, metabolism and tissue sensitivity. Plant Physiology 97: 481-490.

Fett-Neto, A., J. Fett, L. Vieira, G. Pascuali, R. Termignomi \& A. Ferreira. 2001. Distinct effects of auxin on adventitious root development Eucalyptus saligna and Eucalyptus globulus. Tree Physiology 21: 437-461.

Flores, C., A. Cabañas, I. Peñalosa, R. Quintanar, J. Vásquez \& M. UrzúA. 2009. Auxina endógena, AIA-oxidasa y enraizamiento en Vigna radiata L. Wilczek inducida por auxina exógena libre y conjugada. Revista Fitotecnia Mexicana 32(1): 61-66.

FrimL, J. 2003. Auxin transport - zapping the plant. Current Opinion. Plant Biology 6: 7- 12.

Gaspar, M.J., N. Borralho \& A. Lopes. 2005. Comparison between field performance of cuttings and seedlings of Eucalyptus globulus. Annual Science 62: 837-841.

Geiss, G., L. Gutierrez \& C. Bellini. 2009. Adventitious root formation: new insight and perspectives. Annual Plant Reviews 37: 127-156.

Gutiérrez, D., C.H. Ortiz \& A. García y Mendoza. 2008. Medición de fenoles y actividad antioxidante en malezas usadas para alimentación animal. UAQ. México. Pp. 1-5.

Hartmann, H.T., D.E. Kester, F.T. Davies \& R.L. Geneve. 2002. Plant Propagation. Principles and Practices. 8th ed., Prentice Hall, New Jersey. 880 pp. 
Kevers, C., C. Bringaud, J.F. Hausman \& T.H. Gaspar. 1997. Putrescine involvement in the inductive phase of walnut shoots rooting in vitro. Saussurea 28: 47-57.

Koes, R., W. Verweis \& F. Quanttrocchio. 2005. Flavonoides: un modelo colorimétrico de la regulación y la evolución de las vías bioquímicas. Plant Science 10(5): 236-242.

Kozai, T. \& C. Kubota. 2005. Concepts, definitions, ventilation methods, advantages and disanvantages. In: T. Kozai, F. Afreen \& S.M.A Zobayed (eds.), Photoautotrophic (sugar free medium) micropropagation as a new propagation and transplant production system. Pp. 19-30. Springer. Dordrecht.

Latsague, M. \& J. Lara. 2003. Fenoles solubles y su relación con la inhibición de la rizogénesis en estacas de Nothofagus pumilio (Poepp. et Endl.) Krasser. Gayana Botánica (60)2: 85-90.

Littell, R.C., A.G. Milliken, W.W. Stroup, R.D. Wolfinger \& O. SCHABENBERGER. 2006. SAS for Mixed Models, 2nd Edition. SAS Institute Inc., Cary, North Carolina, USA. 519 pp.

Li, S., L Xue, S. Xu, H. Feng \& L. An. 2009. Mediators, genes and signaling in adventitious rooting. Botanical Reviews 75: 230-247.

LiU, J.H. \& D.M REID. 1992. Adventitious rooting in hypocotyls of sunflower (Helianthus annuus) seedlings. IV. The role of changes in endogenous free and conjugated indole-3acetic acid. Physiology Plant 86: 285-292.

LudwiG-MüLLER, J.L. 2000. Indole-3-butyric acid in plant growth and development. Plant Growth Regulation 32: 219-230.

Mankessi, F., A.R. Saya, C. Baptiste, S. Nourissier \& O. MonteuUis. 2009. In vitro rooting of genetically related Eucalyptus urophylla $\times$ Eucalyptus grandis clones in relation to the time spent in culture. Trees 23: 931-940.

Martellet, C. \& A. Fett-Neto. 2005. Role of auxin and its modulators in the adventitious rooting of Eucalyptus species differing in recalcitrance. Plant Growth Regulation 45: 1-10.

Martínez Pastur, G., M.E. Arena, N. Curvetto, D. Zappacosta \& E. Eliasco. 2003. Successive media to improve in vitro rhizogenesis of Nothofagus nervosa (Phil.) Dim. et Mil. New Forests 26: 201-215.

Martínez Pastur, G., M. Arena, L. Hernández, N. Curvetto \& E. Eliasco. 2005. Histological events during in vitro rooting of Nothofagus nervosa (Fagaceae). New Zealand Journal Botanic 43: 61-70.

MetaXas, D.J., T.D. Syros, T. Yupsanis \& A.S. Economou. 2004. Peroxidases during adventitious rooting in cuttings of Arbutus unedo and Taxus baccata as affected by plant genotype and growth regulator treatment. Plant Growth Regulation 44: 257-266.

Murashige, T. \& F. Skoog. 1962. A revised medium for rapid growth and bioassays with tobacco tissue culture. Physiologia Plantarum 15: 473-479.

Murphy, A., W.A. Peer \& L. Taíz. 2000. Regulation of auxin transport by aminopeptidases and endogenous flavonoids. Planta 211: 315-324.

NaG, S., K. SAha \& M.A. Chouthuri. 2001. Role of Auxin and Polyamines in Adventitious Root Formation in Relation to Changes in Compounds Involved in Rooting. Journal Plant Growth Regulation 20: 152- 194.

Naija, S., N. Elloumi, N. Jbir, S. Ammar \& C. Kevers. 2008.
Anatomical and biochemical changes during adventitious rooting of apple rootstocks MM 106 cultured in vitro. Cell Roots Biologies 331: 518-525.

Nordstrom, A.C., A.C. Jacobs \& L. Eliasson. 1991. Effect of exogenous indole-3- acetic acid and indole-3-butyric acid on the internal levels of the respective auxins and their conjugation with aspartic acid during adventitious root formation in pea cuttings. Plant Physiology 96: 856-861.

Pacheco, P., X. Calderón \& A. Vega. 1995. Flavonoids as regulators and markers of root formation by shoots of Eucalyptus globulus in vitro. Plant Peroxidase News 1 (5): 9-12.

Peer, W., A. Bandyopadhyay, J. Blakeslee, S. Makam, R. Chen, P. Masson \& A. Murphy. 2004. Variation in Expression and Protein Localization of the PIN Family of Auxin Exfflux Facilitator Proteins in Flavonoid Mutants with Altered Auxin Transport in Arabidopsis thaliana. The Plant Cell 16: 1898-1911.

Popsilová, J., D. Haisel, H. Synková, J. Catsky, N. Wihelmová, S. Pizaková, D. Prochazková \& F. Sramek. 2000. Photosynthetic pigments and gas exchange during ex vitro acclimatation of tobacco plants as affected by $\mathrm{CO}_{2}$ supply and abscisic acid. Plant Cell Tissue and Organ Culture 61: $125-133$

QAddoury, A. \& M. Amsa. 2004. Effect of exogenous indole butyric acid on root formation and peroxidase and indole3 -acetic acid oxidase activities and phenolic contents in dare palm off-shoots. Botanical Bulletin Academia Sinica 45: 127-131.

Reinhard, D., T. Mandel \& C. Kuhlemeier. 2000. Auxin regulates the initiation and Radial position of Plant Lateral Organs. The Plant Cell 12: 507-518.

Ríos, D., M. Sánchez-Olate, M. Gea, R. Rodríguez. 2002. Nuevos sistemas experimentales para el estudio de la rizogénesis en nogal. Agrociencia 17: 221-228.

Ríos, D., D. Avilés, M. Sánchez-Olate, R. Escobar \& C. Pereira. 2005. Rooting Rate Variation Related to Subculture Number and Diameter of Chestnut Castanea sativa Mill. Microshoots. Agricultura Técnica (Chile) 65(3): 258-264.

Robert-Klever, N., J. Albreechlová, S. Fleig, N. Huck, W. Michalke, E. Wagner, V. Speth, W. Neuhrus \& Ch. Fischer-Iglesias. 2003. Plasma Membrane $\mathrm{H}^{+}$-ATPase Is Involved in Auxin-Mediated Cell Elongation during Wheat Embryo Development. Plant Physiology 131: 1302-1312.

San José, M.C., L. Romero \& L. Janeiro. 2012. Estudio anatómico del desarrollo de raíces adventicias en microestaquillas de Alnus glutinosa (L.) Gaertn. Revista Real Academia Galega de Ciencias 31: 15-26.

Solar, A., M. Colaric, V. Usenik \& F. Stampar. 2006. Seasonal variations of selected flavonoids, phenolic acids and quinons in annual shoots of common walnut (Juglans regia L.). Plant Science 170: 453-461.

Schwambach, J., C. Michels Ruedell, M. Rodrigues De Almeida, R. Penchel, E. Frank De Arau \& A. Fett-Neto. 2008. Adventitious rooting of Eucalyptus globulus x maidennii mini-cuttings derived from mini-stumps grown in sand bed and intermittent flooding trays: a comparative study. New Forests 36: 261-271.

Strader, L., A. Hendrickson, J. Cohen \& B. Bartel. 2010. 
Conversion of Endogenous Indole-3-Butyric Acid to Indole-3-Acetic Acid Drives Cell Expansion in Arabidopsis Seelings. Plant Physiology 153: 1577-1586.

TAYLOR, L.P. \& E. GRoTEWOLD. 2005. Flavonoids as developmental regulators. Current Opinion Plant Biology 8: 317-323.

Teale, W., I. Paponov \& K. Palme. 2006. Auxin in action: signalling, transport and the control of plant growth and development. Nature Review Molecular Cell Biology 7: 847-859.
Takahashi, F., K. Sato-Nara, K. Kobayashi, M. SuzuKi \& H. Suzuki. 2003. Sugar-induced adventitious roots in Arabidopsis seedlings. Journal of Plant Research 116: 83-91.

Valenzuela, S., F. Hevia, M. Tello, L. Minoletti, R. Wilkens \& A. Urbina. 1998. Determinación de ABA en hojas de Eucalyptus globulus a través de HPLC. Agrociencia 14: 29-34.

Woodward, A. \& B. Bartel. 2005. Auxin: Regulation, Action and Interaction. Annals of Botany 95: 707-735.

Recibido: 21.01 .14

Aceptado: 01.10.15 